\title{
A Compact-Type CIP Method for General Korteweg-de Vries Equation
}

\author{
YuFeng Shi, ${ }^{1}$ Biao XU, ${ }^{2}$ and Yan Guo ${ }^{3}$ \\ ${ }^{1}$ School of Electric Power Engineering, China University of Mining and Technology, Xuzhou, Jiangsu 221116, China \\ ${ }^{2}$ School of Mathematical Sciences, Huaibei Normal University, Huaibei, Anhui 235000, China \\ ${ }^{3}$ Department of Mathematics, China University of Mining and Technology, Xuzhou, Jiangsu 221116, China
}

Correspondence should be addressed to Biao XU; xubiao512@163.com

Received 26 April 2014; Revised 13 July 2014; Accepted 15 July 2014; Published 3 August 2014

Academic Editor: Yushun Wang

Copyright (C) 2014 YuFeng Shi et al. This is an open access article distributed under the Creative Commons Attribution License, which permits unrestricted use, distribution, and reproduction in any medium, provided the original work is properly cited.

We proposed a hybrid compact-CIP scheme to solve the Korteweg-de Vries equation. The algorithm is based on classical constrained interpolation profile (CIP) method, which is coupled with high-order compact scheme for the third derivatives in Korteweg-de Vries equation. Several numerical examples are presented to confirm the high resolution of the proposed scheme.

\section{Introduction}

The Korteweg-de Vries (KdV) equation, developed by Korteweg and de Vries [1] in 1895 to model weakly nonlinear waves, has been used in many different fields to model various physical phenomena of interest. In recent years, a number of numerical methods are proposed for solving KdV equations. In [2], Zabusky and Kruskal proposed a finite difference method for KdV equations. A local discontinuous Galerkin method was developed for solving KdV type equations containing third derivative terms in one and two space dimensions in [3]. Numerical solutions for general KdV equation with Crank Nicolson method and B-spline FEM were compared with those obtained with Adomian decomposition method (ADM) in $[4,5]$. Based on the multisymplectic theory, lots of numerical schemes were proposed for KdV equations [6-8]. Ascher and McLachlan developed a simplified 8-point box scheme [9]. By adding an artificial numerical condition to the periodic boundary, Wang et al. derived some new schemes and proved that they were more efficient than the Preissman scheme in [10]. A completely explicit 6-point multisymplectic scheme is derived in [11]. Recently many other numerical methods have been proposed for solving $\mathrm{KdV}$ type equations in [12-18].

In recent years, the less diffusive CIP scheme developed by Takewaki et al. [19] for solving hyperbolic equation has become very popular. However, the original CIP method [1922] utilizing both the point values and its spatial gradients needs auxiliary boundary conditions for the spatial gradient information. Usually, it has to differentiate the equation with spatial variable to get the values of derivation on the node. For the simple case, where the velocity is constant, the procedure is not difficult, but it is not easy for complex equations. In 1992, Lele [23] developed high-order compact (HOC) difference schemes based on implicit interpolations for first and second derivatives. The implicit schemes are very accurate in smooth regions and have spectral-like resolution properties by using the global grid. High-order compact finite difference schemes coupled with high-order low-pass filter are applied to simulate KdV equations in [24].

In this paper, a new numerical scheme based on classical $\mathrm{CIP}$ and HOC schemes is proposed to solve KdV type equations. The new scheme is based on CIP scheme; as a new ingredient, the classical high-order compact scheme [23] is employed to obtain the derivatives rather than differentiate the equation with spatial variable to construct CIP scheme. By comparing with classical compact scheme for solving KdV equations, no filter is used to formulate the present scheme.

The paper is organized as follows. In Section 2, we give a brief description of CIP and high-order compact schemes. The numerical arithmetic of the present scheme is also discussed in the last part of this section. The implementation 
of our present method for $\mathrm{KdV}$ type equations is shown in Section 3, and the capability of the method for nonlinear dispersive equations can be observed from the comparison of numerical solutions with exact solutions. A short discussion for the present method is given in Section 4 .

\section{Descriptions of Methods}

In this paper, we consider the following generalized $\mathrm{KdV}$ equation [25]:

$$
u_{t}+a(u) u_{x}+\varepsilon u_{x x x}=0
$$

The equation can be split into two parts:

$$
\begin{gathered}
u_{t}+a(u) u_{x}=0, \\
u_{t}=-\varepsilon u_{x x x},
\end{gathered}
$$

where $\varepsilon$ are real constants. We only consider the advective phase (2) to review CIP method.

2.1. The CIP Method. The CIP method in [21] uses cubicpolynomial interpolation to get the values of a function on nodes. The time evolution of spatial derivation is also required (4). We differentiate the advective phase of (2) with the spatial variable, and then the equation for derivatives of $u$ can be obtained [26]. Consider

$$
\frac{\partial g}{\partial t}+a(u) \frac{\partial g}{\partial x}=-g \frac{\partial a(u)}{\partial x},
$$

where $g=\partial u / \partial x$ stands for the spatial derivatives of $u$. The computational domain $[a, b]$ can be divided into $N$ cells, and the cells are denoted by $I_{i}=\left[x_{i}, x_{i+1}\right]$. We only consider a uniform grid and the size of the cell by $\Delta x=(b-a) / N$. The cubic polynomial and the first-order derivative at the $n$th step can be written as

$$
\begin{gathered}
U_{i}^{n}(x)=a_{i} X^{3}+b_{i} X^{2}+c_{i} X+d_{i}, \\
U_{i}^{\prime n}(x)=3 a_{i} X^{2}+2 b_{i} X+c_{i},
\end{gathered}
$$

where $X=x-x_{i}$, and coefficients $a_{i}, b_{i}, c_{i}$, and $d_{i}$ will be obtained with the following constrains:

$$
\begin{aligned}
U_{i}^{n}\left(x_{i}\right) & =u_{i}^{n}, & U_{i}^{n}\left(x_{i u p}\right) & =u_{i u p}^{n}, \\
U_{i}^{\prime n}\left(x_{i}\right) & =g_{i}^{n}, & U_{i}^{\prime n}\left(x_{i u p}\right) & =g_{i u p}^{n},
\end{aligned}
$$

where iup $=i-\operatorname{sgn}\left(a\left(u_{i}\right)\right)$, the sign $\operatorname{sgn}\left(a\left(u_{i}\right)\right)$ stands for the sign of $a\left(u_{i}\right)$. Then, the coefficients of the cubic polynomial are given as follows:

$$
\begin{gathered}
a_{i}=\frac{g_{i}^{n}+g_{i u p}^{n}}{\Delta x_{i}^{2}}+\frac{2\left(u_{i}^{n}-u_{i u p}^{n}\right)}{\Delta x_{i}^{3}}, \\
b_{i}=\frac{3\left(u_{i u p}^{n}-u_{i}^{n}\right)}{\Delta x_{i}^{2}}-\frac{2 g_{i}^{n}+g_{i u p}^{n}}{\Delta x_{i}}, \\
c_{i}=g_{i}^{n}, \quad d_{i}=u_{i}^{n},
\end{gathered}
$$

where $\Delta x_{i}=x_{i u p}-x_{i}$. Thus, the values of $u$ and $g$ at the $(n+1)$ th step can be obtained as follows:

$$
\begin{aligned}
& u_{i}^{n+1}=U_{i}^{n}\left(x_{i}-a\left(u_{i}\right) \Delta t\right), \\
& g_{i}^{n+1}=U_{i}^{\prime n}\left(x_{i}-a\left(u_{i}\right) \Delta t\right) .
\end{aligned}
$$

We define $\xi_{i}=-a\left(u_{i}\right) \Delta t$, and then the formulas are rewritten as

$$
\begin{gathered}
u_{i}^{n+1}=a_{i} \xi_{i}^{3}+b_{i} \xi_{i}^{2}+g_{i}^{n} \xi_{i}+u_{i}^{n}, \\
g_{i}^{n+1}=3 a_{i} \xi_{i}^{n}+2 b_{i} \xi_{i}^{n}+g_{i}^{n} .
\end{gathered}
$$

It can be seen that only two points are used in CIP schemes to get $u_{i}^{n+1}$. Then, the advantages of this method can be shown while the computational boundary is complex since less boundary points need to be handled. The CIP method uses only two neighboring stencils but maintains third-order accuracy. In this sense, high order accuracy is obtained though less computational stencils are used. More details for advantages of the CIP schemes can be found in [26].

2.2. High-Order Compact Scheme. A series finite difference scheme to evaluate the spatial derivatives is presented in [23]. The finite difference approximation to the derivative of the function is expressed as a linear combination of the given function values, and then the derivatives of the function are obtained by solving a tridiagonal or pentadiagonal system. Formulas for the first-order and the third-order derivatives are reviewed as below. More results for the approximation to derivatives can be found in $[23,27]$.

2.2.1. The Derivatives at Interior Nodes. In this paper, we consider the KdV equation on a uniform mesh, the spatial variable at the nodes is $x_{i}=i \times h$ for $0 \leq i \leq N$ and the functions and the derivatives are denoted by $u_{i}, u_{i}^{\prime}$. The firstorder derivatives for interior nodes are derived by writing approximations of the form [23]. Consider

$$
\begin{aligned}
u_{i}^{\prime}+\alpha & \left(u_{i-1}^{\prime}+u_{i+1}^{\prime}\right)+\beta\left(u_{i-2}^{\prime}+u_{i+2}^{\prime}\right) \\
& =c \frac{u_{i+3}-u_{i-3}}{6 h}+b \frac{u_{i+2}-u_{i-2}}{4 h}+a \frac{u_{i+1}-u_{i-1}}{2 h} .
\end{aligned}
$$

If the schemes are restricted to $\beta \geq 0$ and $c=0$, a oneparameter $\alpha$-family of fourth-order tridiagonal scheme is obtained. Consider

$$
\begin{gathered}
\beta=0, \quad c=0, \quad a=\frac{2}{3}(\alpha+2), \\
b=\frac{1}{3}(4 \alpha-1) .
\end{gathered}
$$

A simple sixth-order tridiagonal scheme for first-order derivatives is given with $\alpha=1 / 3, \beta=0, c=0, a=14 / 9$, and $b=1 / 9$ :

$$
u_{i}^{\prime}+\frac{1}{3}\left(u_{i-1}^{\prime}+u_{i+1}^{\prime}\right)=\frac{14}{9} \frac{u_{i+1}-u_{i-1}}{2 h}+\frac{1}{9} \frac{u_{i+2}-u_{i-2}}{2 h} .
$$


For the third derivatives at interior nodes, the following formula is given in [23]:

$$
\begin{aligned}
\alpha\left(u_{i-1}^{\prime \prime \prime}+u_{i+1}^{\prime \prime \prime}\right)+u_{i}^{\prime \prime \prime}= & b \frac{u_{i+3}-3 u_{i+1}+3 u_{i-1}-u_{i-3}}{8 h^{3}} \\
& +a \frac{u_{i+2}-2 u_{i+1}+2 u_{i-1}-u_{i-2}}{2 h^{3}} .
\end{aligned}
$$

The fourth-order tridiagonal schemes can be obtained with the coefficients $a=2$ and $b=2 \alpha-1$. The compact tridiagonal scheme is given with $\alpha=1 / 2, a=2$, and $b=0$. And the sixthorder tridiagonal scheme is given with $\alpha=7 / 16, a=2$, and $b=-1 / 8$.

2.2.2. Nonperiodic Boundaries. For those near boundary nodes, approximation formulas for the first-order derivatives of nonperiodic boundary problems are given by one-side formulation as follows [23]:

$$
\begin{gathered}
u_{1}^{\prime}+\alpha u_{2}^{\prime}=\frac{1}{h}\left(a u_{1}+b u_{2}+c u_{3}+d u_{4}\right) \\
u_{N}^{\prime}+\alpha u_{N-1}^{\prime}=-\frac{1}{h}\left(a u_{N}+b u_{N-1}+c u_{N-2}+d u_{N-3}\right) .
\end{gathered}
$$

The coefficients for schemes of the third and fourth order are given by

Third order:

$$
\begin{aligned}
& a=-\frac{11+2 \alpha}{6}, \quad b=\frac{6-\alpha}{2}, \\
& c=\frac{2 \alpha-3}{2}, \quad d=\frac{2-\alpha}{6},
\end{aligned}
$$

Fourth order:

$$
\begin{array}{ll}
\alpha=3, & a=-\frac{17}{6}, \quad b=\frac{3}{2}, \\
c=\frac{3}{2}, & d=-\frac{1}{6} .
\end{array}
$$

The sixth-order scheme also be given, for those near boundary nodes, six order approximation formulas for the firstorder derivatives can be written as follows:

$$
\begin{aligned}
& u_{1}^{\prime}+\alpha u_{2}^{\prime} \\
& \quad=\frac{1}{h}\left(a_{1} u_{1}+a_{2} u_{2}+a_{3} u_{3}+a_{4} u_{4}+a_{5} u_{5}+a_{6} u_{6}\right),
\end{aligned}
$$

where

$$
\begin{aligned}
& \alpha=5, \quad a_{1}=-\frac{197}{60}, \quad a_{2}=-\frac{12}{5}, \quad a_{3}=5, \\
& a_{4}=-\frac{5}{3}, \quad a_{5}=\frac{5}{12}, \quad a_{6}=-\frac{1}{20} .
\end{aligned}
$$

For the second point, the formula is

$$
\begin{aligned}
\alpha u_{1}^{\prime}+ & u_{2}^{\prime}+\alpha u_{3}^{\prime} \\
& =\frac{1}{h}\left(b_{1} u_{1}+b_{2} u_{2}+b_{3} u_{3}+b_{4} u_{4}+b_{5} u_{5}+b_{6} u_{6}\right),
\end{aligned}
$$

where

$$
\begin{aligned}
& \alpha=\frac{2}{11}, \quad b_{1}=-\frac{20}{33}, \quad b_{2}=-\frac{35}{132}, \quad b_{3}=\frac{34}{33}, \\
& b_{4}=-\frac{7}{33}, \quad b_{5}=\frac{2}{33}, \quad b_{6}=-\frac{1}{132} \text {. }
\end{aligned}
$$

The dissymmetry condition is used for the $N$ th and $(N-1)$ th points.

2.3. The Present Compact-Type CIP Method. In this section, a new compact-type CIP scheme is proposed for (1). For simplicity, the following equation is considered to introduce the method. Consider

$$
u_{t}+\alpha u u_{x}-\delta u_{x x x}=0
$$

where $\alpha$ and $\delta$ are constants. We split the solution of equation into two phases:

$$
\begin{gathered}
\frac{\partial u}{\partial t}+\alpha u \frac{\partial u}{\partial x}=0 \\
\frac{\partial u}{\partial t}=\delta u_{x x x} .
\end{gathered}
$$

We consider a 1D mesh, consisting of $(N+1)$ points: $x_{0}$, $x_{1}, x_{2}, \ldots, x_{N-1}, x_{N}$; the values of $u$ on these nodes at the $n$th step are denoted by $u_{0}^{n}, u_{1}^{n} \ldots, u_{N-1}^{n}, u_{N}^{n}$. At first, (21) is considered and CIP method is applied to the equation. The cubic polynomial at the $n$th time stage is

$$
U_{i}^{n}(X)=a_{i}^{n} X^{3}+b_{i}^{n} X^{2}+c_{i}^{n} X+u_{i}^{n},
$$

where $X=x_{i}-x$ and the coefficient $a_{i}^{n}, b_{i}^{n}$, and $c_{i}^{n}$ are given by (7), where $a(u)=\alpha u$ and $u_{i}^{\prime n}$ denote the derivative of $u_{i}^{n}$ at the $i$ th node. The predictor-corrector scheme is employed to calculate the value $u^{*}$.

In the present method, the values $u_{i}^{\prime n}, 0 \leq i \leq N$ are expressed as a linear combination of the given values $u_{i}^{n}$, $0 \leq i \leq N$. On the other hand, the HOC method is employed to evaluate the derivatives $u_{i}^{\prime n}, 0 \leq i \leq N$. A simple sixthorder tridiagonal scheme for interior points and boundary points is used in this paper.

Temporal discretization for (22) can be solved by using third-order Runge-Kutta method:

$$
\begin{gathered}
u^{(1)}=u^{n}+\delta t u_{x x x}^{*}, \\
u^{(2)}=\frac{3}{4} u^{n}+\frac{1}{4} u^{(1)}+\frac{1}{4} \delta t u_{x x x}^{(1)}, \\
u^{n+1}=\frac{1}{3} u^{n}+\frac{2}{3} u^{(2)}+\frac{2}{3} \delta t u_{x x x}^{(2)} .
\end{gathered}
$$

The HOC scheme (13) is used to solve the third derivatives $u_{x x x}$ in (24). The sixth-order tridiagonal scheme with the periodic boundary condition is used in this paper.

The essential ingredients of the computational algorithm for (20) are presented below. Suppose we have got the values $u_{i}^{n}$. The values $u_{i}^{n+1}$ are given as follows. 
TABLE 1: Numerical errors and orders of CIP-HOC method for the KdV equation (29).

\begin{tabular}{lcccccccc}
\hline$N$ & $L_{\infty}$ error & $L_{\infty}$ order & $L_{2}$ error & $L_{2}$ order & $I_{1}$ & $I_{2}$ & $I_{3}$ \\
\hline 20 & $5.72 E-03$ & & $9.94 E-03$ & & 1.9968246 & 0.6627258 & 0.5745942 & $6.25 E-05$ \\
40 & $1.32 E-03$ & 2.12 & $1.89 E-03$ & 2.39 & 1.9996543 & 0.6663460 & 0.5774709 & $2.50 E-04$ \\
80 & $2.11 E-04$ & 2.65 & $2.85 E-04$ & 2.73 & 1.9999840 & 0.6666518 & 0.5777566 & $2.50 E-03$ \\
160 & $2.77 E-05$ & 2.93 & $3.61 E-05$ & 2.98 & 1.9999984 & 0.6666662 & 0.5777761 & $3.13 E-02$ \\
320 & $3.49 E-06$ & 2.99 & $4.54 E-06$ & 2.99 & 1.9999988 & 0.6666667 & 0.5777776 & $6.09 E-01$ \\
\hline
\end{tabular}

TABLE 2: Numerical errors and invariants with $N=100$ at different times.

\begin{tabular}{lccccc}
\hline Time & $L_{\infty}$ error & $L_{2}$ error & $I_{1}$ & $I_{2}$ \\
\hline 0.10 & $1.09 E-04$ & $1.47 E-04$ & 1.9999939 & 0.6666616 & 0.5777688 \\
0.20 & $1.65 E-04$ & $2.47 E-04$ & 1.9999888 & 0.6666565 & 0.5777615 \\
0.30 & $1.98 E-04$ & $3.14 E-04$ & 1.9999830 & 0.6666515 & 0.5777556 \\
0.40 & $2.14 E-04$ & $3.61 E-04$ & 1.9999775 & 0.6666464 & 0.5777505 \\
0.50 & $2.28 E-04$ & $3.94 E-04$ & 1.9999760 & 0.6666413 & 0.5777461 \\
0.60 & $2.41 E-04$ & $4.18 E-04$ & 1.9999660 & 0.6666363 & 0.5777421 \\
0.70 & $2.46 E-04$ & $4.37 E-04$ & 1.9999590 & 0.6666312 & 0.5777383 \\
0.80 & $2.42 E-04$ & $4.52 E-04$ & 1.9999651 & 0.6666211 & 0.5777347 \\
0.90 & $2.51 E-04$ & $4.63 E-04$ & 1.9999605 & 0.6666161 & 0.57777277 \\
1.00 & $2.59 E-04$ & $4.73 E-04$ & 1.9999441 & & \\
\hline
\end{tabular}

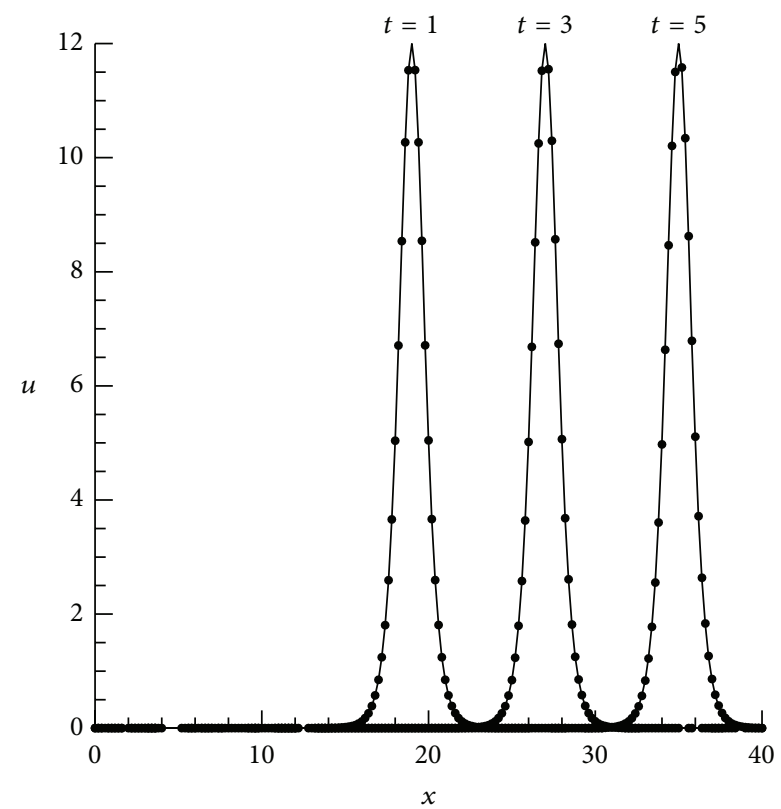

FIGURE 1: Numerical and analytical solutions for equation $u_{t}+u u_{x}+$ $u_{x x x}=0$ of Example 2 at various time stages.

(1) CIP method is used to obtain $u^{*}$.

(a) The values of the first derivative on the all nodes are obtained by using the HOC scheme (12).

(b) Predictor-corrector CIP scheme is as follows: (i) predictor step:

$$
\begin{aligned}
u_{i}^{* *} & =U_{i}^{n}\left(x_{i}-\alpha u_{i}^{n} \Delta t\right) \\
& =a_{i}^{n} \xi_{i}^{3}+b_{i}^{n} \xi_{i}^{2}+c_{i}^{n} \xi_{i}+u_{i}^{n},
\end{aligned}
$$

where $\xi_{i}=-\alpha u_{i}^{n} \Delta t$. We also get $u^{* * *}$ at the $(n+1 / 2)$ th time stage by using linear interpolation or QUICK scheme based on the value $u_{i}^{n}$;

(ii) corrector step (CIP method):

$$
\begin{aligned}
\widehat{u}_{i}^{*} & =U_{i}^{n}\left(x_{i}-\alpha u_{i}^{\diamond} \Delta t\right) \\
& =a_{i}^{n} \xi_{i}^{3}+b_{i}^{n} \xi_{i}^{2}+c_{i}^{n} \xi_{i}+u_{i}^{n},
\end{aligned}
$$

where $u^{\diamond}=(1 / 2)\left(u^{* *}+u^{* * *}\right)$;

(iii) the predictor and corrector step are employed again to get $u^{*}$.

(2) HOC scheme and Runge-Kutta method for (22) are as follows.

(a) The HOC scheme (13) is used to obtain thirdorder derivatives.

(b) Temporal discretization for (22) can be solved by using third-order Runge-Kutta method.

The predictor-corrector scheme is an important step in the present method. Periodic boundary condition is applied to $(22)$. 

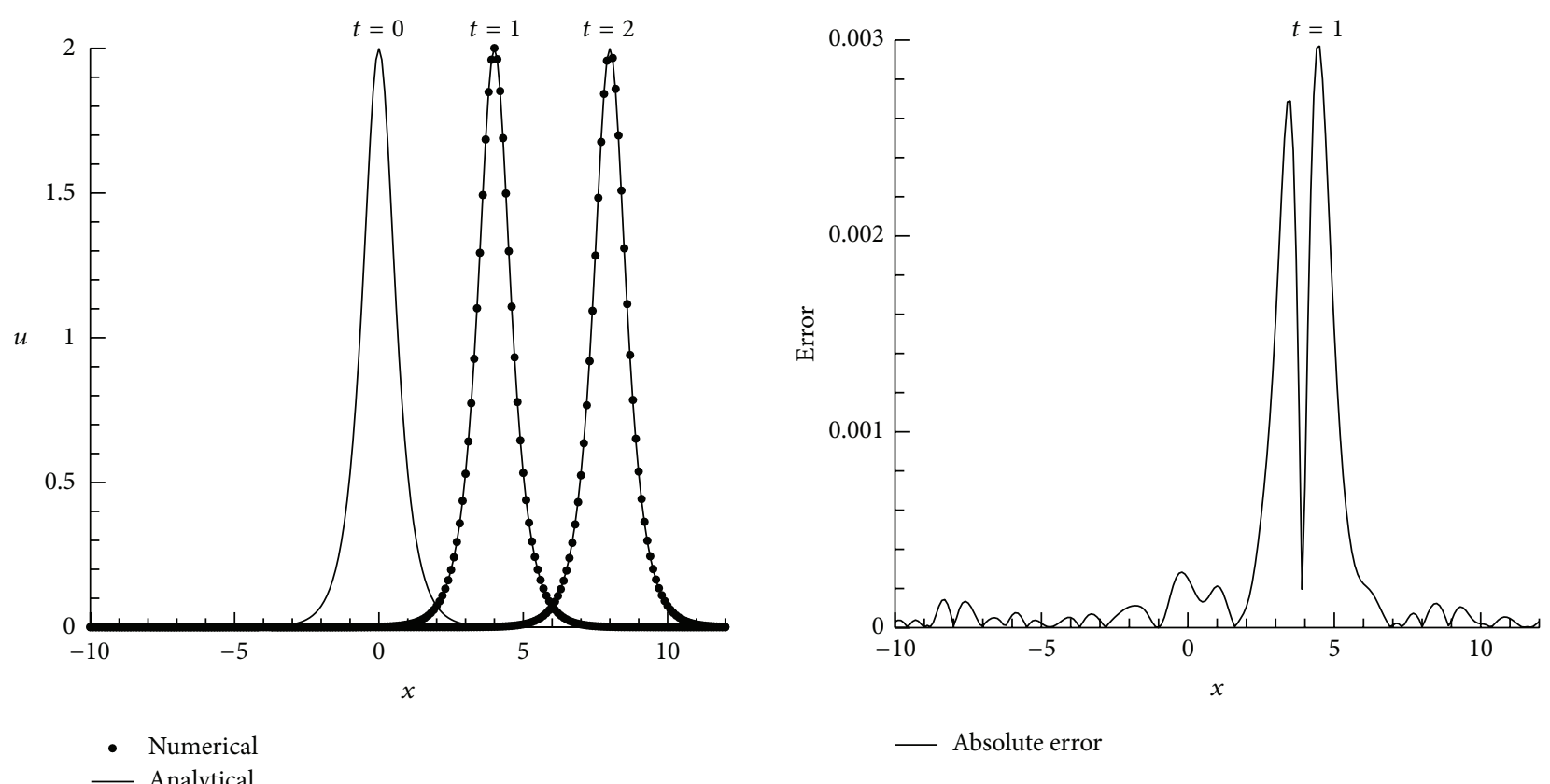

FIGURE 2: Numerical and analytical solutions (line) for equation $u_{t}+6 u^{2} u_{x}+u_{x x x}=0$ of Example 3 at various time stages and the absolute error for $t=1$.

\section{Numerical Results}

In this section, some numerical tests for $\mathrm{KdV}$ and general $\mathrm{KdV}$ equations are carried out. The discrete $L_{2}$ and $L_{\infty}$ error norms are defined as follows:

$$
\begin{gathered}
\left\|e_{n}\right\|_{L_{\infty}}=\max _{0 \leq j \leq N}\left|u_{j}-\tilde{u}_{j}\right|, \\
\left\|e_{n}\right\|_{L_{2}}=\left(\sum_{j=0}^{N}\left|u_{j}-\tilde{u}_{j}\right|^{2} \Delta x\right)^{1 / 2},
\end{gathered}
$$

where $u$ and $\tilde{u}$ are exact and numerical solution, respectively. For KdV equations, there are an infinite number of conservation laws [28]. We will focus our analysis on the following three conservation laws:

$$
\begin{aligned}
& I_{1}=\int_{a}^{b} u(x, t) d x, \quad I_{2}=\int_{a}^{b} u(x, t)^{2} d x, \\
& I_{3}=\int_{a}^{b}\left(u(x, t)^{2}-\frac{1}{3} u(x, t)^{3}\right) d x,
\end{aligned}
$$

where $I_{1}, I_{2}$, and $I_{3}$ represent mass, momentum, and energy.

The nonperiodic boundary formulation is applied to (21) (HOC approximation formulas for the third-order derivatives are used) and periodic boundary conditions for thirdorder derivatives are used in the following examples.

Example 1. In this example, we consider the following classical KdV equation:

$$
u_{t}+6 u u_{x}+u_{x x x}=0, \quad-15 \leq x \leq 15 .
$$

TABLE 3: Error norms at different time stages for equation $u_{t}+$ $6 u^{2} u_{x}+u_{x x x}=0$, with $u(x, 0)=\sqrt{c} \operatorname{sech}(\sqrt{c} x),-10 \leq x \leq 12, c=$ 0.5. $d x=0.01$, and $d t=0.05 \times d x^{3}$.

\begin{tabular}{lcc}
\hline$T$ & $L_{\infty}$ & $L_{2}$ \\
\hline 0.1 & $5.354322207020701 E-004$ & $4.972071746712235 E-004$ \\
0.5 & $6.080056468253936 E-004$ & $7.277368149932270 E-004$ \\
1.0 & $2.970167786819244 E-003$ & $3.320246422392297 E-003$ \\
\hline
\end{tabular}

The analytical solution for (29) is

$$
u(x, t)=0.5 \operatorname{sech}^{2}(0.5(x-t))
$$

The time-steps are set by the relation $\Delta t=C(\Delta x)^{3}$. The $L_{2}$ and $L_{\infty}$ errors, orders, invariants, and time costs at time $T=0.1$ are illustrated in Table 2 . It can be observed that the proposed scheme is third-order accurate in the spatial dimension. It is well known that high-order TVD RungeKutta methods suffer from small time-step restrictions. In this case, we observe that numerical errors are still not dominated by the spatial discretization with the relation $\Delta t=$ $C(\Delta x)^{3}$.

Table 1 indicates $L_{2}$ and $L_{\infty}$ errors and invariants with $N=100$ at time $T=0.1,0.2, \ldots, 0.9$. The present method can also be shown to have the conservative property.

Example 2. In this example, we consider the following classical KdV equation:

$$
u_{t}+u u_{x}+u_{x x x}=0, \quad 0 \leq x \leq 40
$$


TABLE 4: Error norms at different time stages for equation $u_{t}+6 u u_{x}+u_{x x x}=0$, with $u(x, 0)=(c / 2) \operatorname{sech}^{2}((\sqrt{c} / 2) x-7), c=0.5 . d x=0.2$, and $d t=0.1 \times d x^{3}$.

\begin{tabular}{lccrr}
\hline$T$ & \multicolumn{2}{c}{ Present } & \multicolumn{2}{c}{ Dehghan and Shokri [14] } \\
\hline 1.0 & $L_{\infty}$ & $L_{2}$ & $L_{\infty}$ \\
2.0 & $1.9363 E-005$ & $2.7752 E-005$ & $1.8048 E-005$ & $6.2366 E-005$ \\
3.0 & $2.8040 E-005$ & $4.4840 E-005$ & $3.0373 E-005$ & $1.1264 E-005$ \\
4.0 & $3.5184 E-005$ & $6.2377 E-005$ & $4.0088 E-005$ & $1.5537 E-005$ \\
5.0 & $4.2136 E-005$ & $8.2619 E-005$ & $4.8347 E-005$ & $1.9400 E-005$ \\
\hline
\end{tabular}
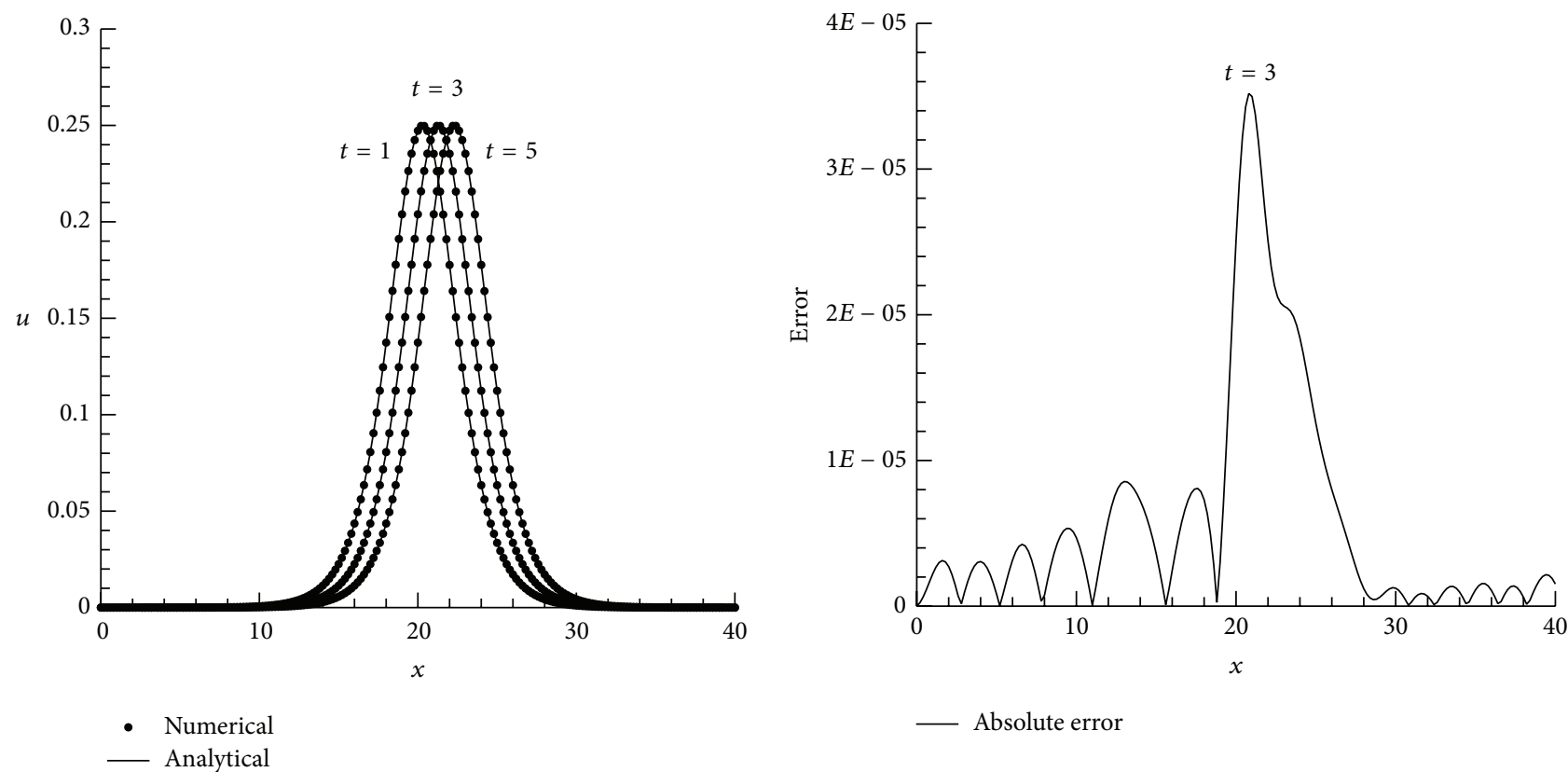

FIGURE 3: Numerical and analytical solutions for equation $u_{t}+6 u u_{x}+u_{x x x}=0$ with initial condition $u(x, 0)=(c / 2) \operatorname{sech}((\sqrt{c} / 2) x-7), 0 \leq$ $x \leq 40, c=0.5$ of Example 3 at various time stages and the absolute error for $t=3$.

with initial condition

$$
u(x, 0)=\frac{12}{\cosh (x-15)^{2}}, \quad 0 \leq x \leq 40 .
$$

The numerical solutions are obtained with $d x=0.2$ and $d t=0.0001$; numerical and exact solutions at times $t=1,3,5$ are presented in Figure 1; the figure shows that the numerical dissipation of soliton is very small.

Example 3. The general $\mathrm{KdV}$ equation is presented by the following equation [29]:

$$
u_{t}+6 u^{p} u_{x}+u_{x x x}=0
$$

Consider the initial value problem associated with (33) using the initial condition for $p=2$ :

$$
u(x, 0)=\sqrt{c} \operatorname{sech}(\sqrt{c} x), \quad-10 \leq x \leq 12,
$$

where $c=4$. The numerical solutions for $p=2$ are obtained with $d x=0.1$ and $d t=0.05 \times d x^{3}$ for $p=2$. The progress of the numerical and analytical solutions at times $t=1,2$ and the absolute error at $t=1$ are shown in Figure 2 . The $L_{\infty}$ and $L_{2}$ error estimates for the case $p=2$ are given in Table 3 .

For the case $p=1$ with the initial condition [14]

$$
u(x, 0)=\frac{c}{2} \operatorname{sech}^{2}\left(\frac{\sqrt{c}}{2} x-7\right), \quad 0 \leq x \leq 40,
$$

and the analytical solution is

$$
u(x, t)=\frac{c}{2} \operatorname{sech}^{2}\left(\frac{\sqrt{c}}{2}(x-c t)-7\right) .
$$

The $L_{\infty}$ and $L_{2}$ error estimates with $d x=0.2$ are given in Table 4 for $t=1,2,3,4,5$, from which it is not difficult to see that the present results are comparable with those present in [14]. The numerical and the analytical solutions at times $t=$ $1,3,5$ and the absolute error for $t=3$ are shown in Figure 3. 


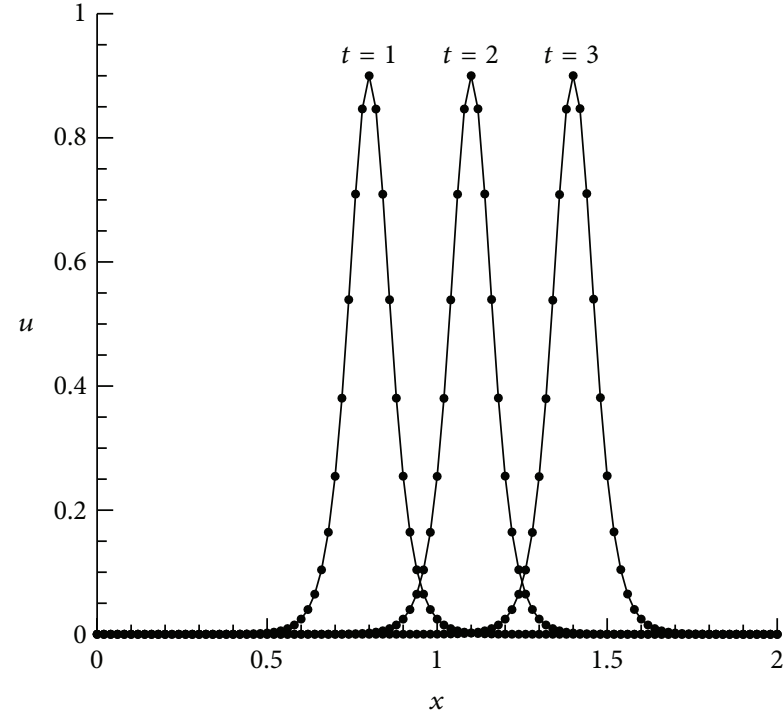

- Numerical Analytical

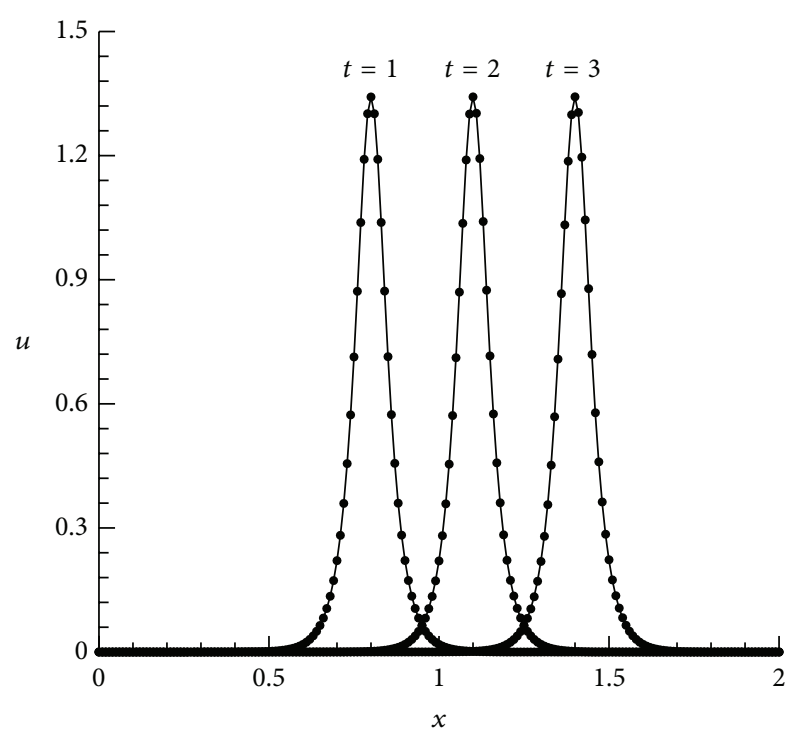

- Numerical Analytical

(a) $u_{t}+u u_{x}+\varepsilon u_{x x x}=0$

(b) $u_{t}+u^{2} u_{x}+\varepsilon u_{x x x}=0$

FIGURE 4: Numerical and analytical solutions for equation $u_{t}+u^{2} u_{x}+\varepsilon u_{x x x}=0$ and $u_{t}+u^{2} u_{x}+\varepsilon u_{x x x}=0$ of Example 4 at various time stages.

TABLE 5: Error norms at different time stages for equation $u_{t}+u u_{x}+\varepsilon u_{x x x}=0$, with initial condition $(38)$ for $n=1, d x=0.02$, and $d t=0.25 \times d x^{2}$.

\begin{tabular}{lccrr}
\hline$T$ & \multicolumn{2}{c}{ Present } & \multicolumn{2}{c}{ Dehghan and Shokri [14] } \\
\hline 0.5 & $L_{\infty}$ & $L_{2}$ & $L_{\infty}$ & $2.5286 E-003$ \\
1.0 & $1.7139 E-004$ & $5.4536 E-005$ & $7.2329 E-004$ & $6.2172 E-003$ \\
1.5 & $1.6824 E-004$ & $5.3897 E-005$ & $1.7957 E-004$ & $1.3010 E-002$ \\
2.0 & $1.9094 E-004$ & $6.9749 E-005$ & $3.8906 E-003$ & $2.2965 E-002$ \\
\hline
\end{tabular}

TABLE 6: Error norms at different time stages for equation $u_{t}+u^{2} u_{x}+$ $\varepsilon u_{x x x}=0$, with initial condition (38) for $n=2, d x=0.01$, and $d t=0.5 \times d x^{2}$.

\begin{tabular}{lcc}
\hline$T$ & $L_{\infty}$ & $L_{2}$ \\
\hline 0.5 & $3.252960639366087 E-004$ & $9.332884478549439 E-005$ \\
1.0 & $3.962848426597443 E-004$ & $1.333167429458641 E-004$ \\
1.5 & $1.160144038488964 E-003$ & $3.754080717733928 E-004$ \\
2.0 & $2.442770975886521 E-003$ & $7.776565570880883 E-004$ \\
\hline
\end{tabular}

Example 4. In this example, another type of general $\mathrm{KdV}$ equation is considered:

$$
u_{t}+u^{n} u_{x}+\varepsilon u_{x x x}=0
$$

with the initial value problem [30]

$$
u(x, 0)=\left(\frac{c(n+1)(n+2)}{2}\right)^{1 / n} \operatorname{sech}^{2 / n}\left(\frac{n}{2} \sqrt{\frac{c}{\varepsilon}}\left(x-x_{0}\right)\right),
$$

where $c=0.3, \varepsilon=0.000484$. The single soliton solutions for $n=1$ are computed in $x \in[0,2]$ with space step $d x=0.02$ and time step $d t=0.25 \times d x^{2}$ and are shown in Figure 4 . The numerical and analytical solutions for $n=2$ with $d x=0.01$ and $d t=0.5 \times d x^{2}$ are shown in Figure 4 . The $L_{\infty}$ and $L_{2}$ error estimates for $n=1$ at times $t=0.5,1,1.5,2$ are given in Table 5. We can observe that the present results are slightly more accurate than those present in [14]. Table 6 shows the $L_{\infty}$ and $L_{2}$ error estimates for the case of $n=2$.

Example 5. We also consider the equation [3]

$$
u_{t}+u u_{x}+\varepsilon u_{x x x}=0, \quad 0 \leq x \leq 2 .
$$

The double soliton collision case has the initial condition

$$
\begin{aligned}
u(x, 0)= & 3 c_{1} \operatorname{sech}^{2}\left(k_{1}\left(x-x_{1}\right)\right) \\
& +3 c_{2} \operatorname{sech}^{2}\left(k_{2}\left(x-x_{2}\right)\right),
\end{aligned}
$$

where $c_{1}=0.3, c_{2}=0.1, x_{1}=0.4, x_{2}=0.8, k_{i}=0.5 \sqrt{c_{i} / \varepsilon}$, and $\varepsilon=4.84 \times 10^{-4}$. The solution is computed in $x \in[0,2]$ and is shown in Figure 5. We can observe that nonoscillate numerical solutions can be obtained by using the present method. 

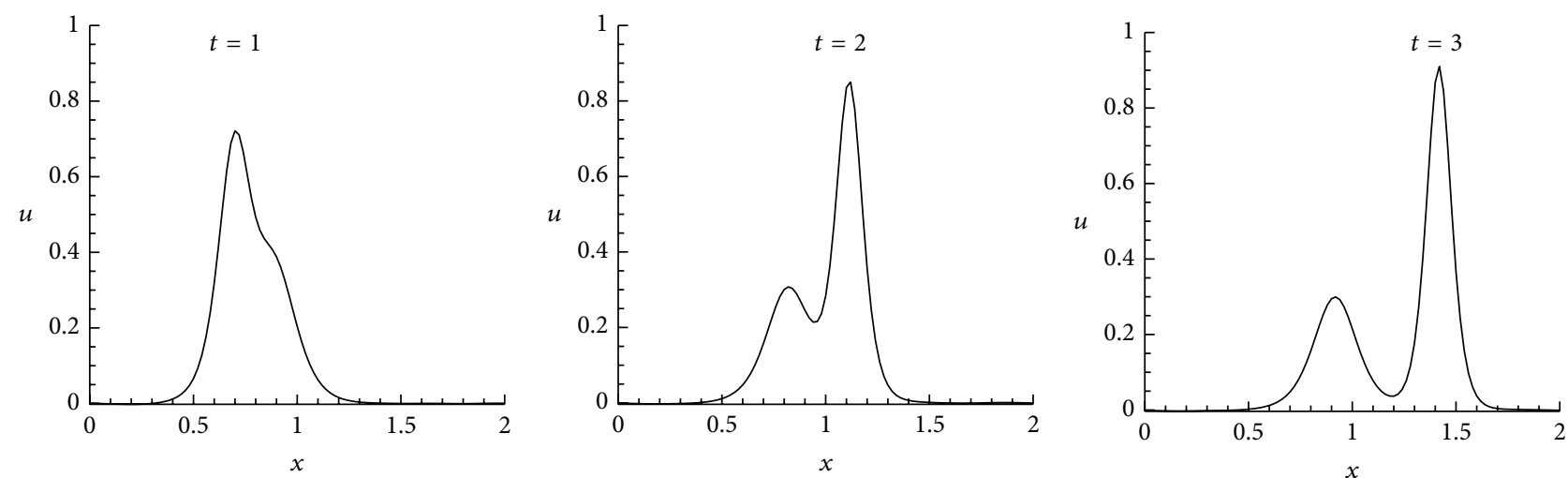

FIGURE 5: Double soliton solutions for equation $u_{t}+u u_{x}+\varepsilon u_{x x x}=0$ of Example 5 at various time stages.
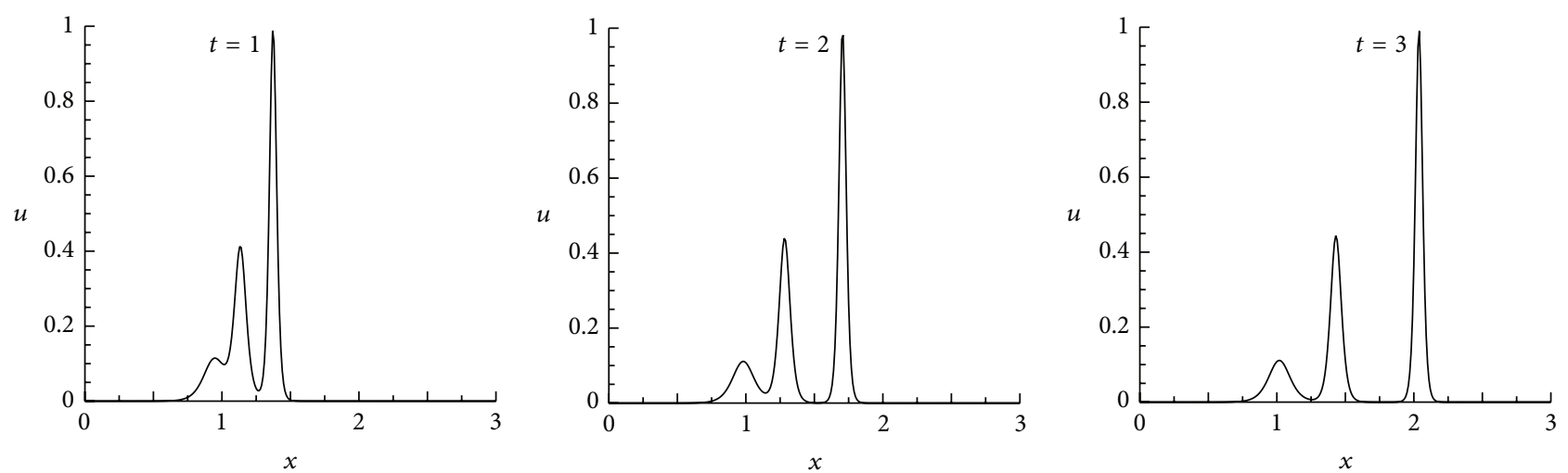

FIgURE 6: Triple soliton solutions for equation $u_{t}+u u_{x}+\varepsilon u_{x x x}=0$ of Example 3 at various time stages.

The triple soliton collision case has the initial condition

$$
u(x, 0)=\frac{2}{3} \operatorname{sech}^{2}\left(\frac{x-1}{\sqrt{108 \varepsilon}}\right)
$$

with $\varepsilon=10^{-4}[3]$. The numerical solution is computed in $x \in[0,3]$ and is shown in Figure 6. From the figure we can conclude that the numerical algorithm captures the numerical solutions without oscillations.

\section{Conclusions}

In this paper, we have presented a new scheme based on the traditional CIP and HOC scheme. A conclusion can be drawn from the comparison between the numerical and the exact solutions that the present compact-type CIP method provides highly accurate numerical solutions of KdV type equations. The numerical results also show that the present method works well for some nonlinear problems.

\section{Conflict of Interests}

The authors declare that there is no conflict of interests regarding the publication of this paper.

\section{Acknowledgments}

The work was partly supported by the Fundamental Research Funds for the Central Universities (2010QNA39, 2012QNB07) and the Natural Science Foundation of Anhui Province (1408085MA14).

\section{References}

[1] D. J. Korteweg and G. de Vries, "XLI. On the change of form of long waves advancing in a rectangular canal, and on a new type of long stationary waves," Philosophical Magazine Series 5, vol. 39, no. 240, pp. 422-443, 1895.

[2] N. J. Zabusky and M. D. Kruskal, "Interaction of "solitons" in a collisionless plasma and the recurrence of initial states," Physical Review Letters, vol. 15, no. 6, pp. 240-243, 1965.

[3] J. Yan and C.-W. Shu, "A local discontinuous Galerkin method for KdV type equations," SIAM Journal on Numerical Analysis, vol. 40, no. 2, pp. 769-791, 2002.

[4] M. A. Helal and M. S. Mehanna, "A comparison between two different methods for solving KdV-Burgers equation," Chaos, Solitons and Fractals, vol. 28, no. 2, pp. 320-326, 2006.

[5] T. Geyikli and D. Kaya, "Comparison of the solutions obtained by B-spline FEM and ADM of KdV equation," Applied Mathematics and Computation, vol. 169, no. 1, pp. 146-156, 2005. 
[6] Z.-Q. Lv, M. Xue, and Y.-S. Wang, "A new multi-symplectic scheme for the KdV equation," Chinese Physics Letters, vol. 28, no. 6, Article ID 060205, 2011.

[7] H.-P. Wang, Y.-S. Wang, and Y.-Y. Hu, "An explicit scheme for the KdV equation," Chinese Physics Letters, vol. 25, no. 7, pp. 2335-2338, 2008.

[8] P. F. Zhao and M. Z. Qin, "Multisymplectic geometry and multisymplectic preissmann scheme for the KdV equation," Journal of Physics A: Mathematical and General, vol. 33, no. 18, pp. 3613-3626, 2000.

[9] U. M. Ascher and R. I. McLachlan, "Multisymplectic box schemes and the Korteweg-de Vries equation," Applied Numerical Mathematics, vol. 48, no. 3-4, pp. 255-269, 2004.

[10] Y. Wang, B. Wang, and M. Qin, "Numerical implementation of the multisymplectic Preissman scheme and its equivalent schemes," Applied Mathematics and Computation, vol. 149, no. 2, pp. 299-326, 2004.

[11] Y. Wang, B. Wang, and X. Chen, "Multisymplectic Euler box scheme for the KdV equation," Chinese Physics Letters, vol. 24, no. 2, article 312, 2007.

[12] E. N. Aksan and A. Özdeş, "Numerical solution of Kortewegde Vries equation by Galerkin B-spline finite element method," Applied Mathematics and Computation, vol. 175, no. 2, pp. 12561265, 2006.

[13] I. Dağ and Y. Dereli, "Numerical solutions of KdV equation using radial basis functions," Applied Mathematical Modelling, vol. 32, no. 4, pp. 535-546, 2008.

[14] M. Dehghan and A. Shokri, "A numerical method for KdV equation using collocation and radial basis functions," Nonlinear Dynamics. An International Journal of Nonlinear Dynamics and Chaos in Engineering Systems, vol. 50, no. 1-2, pp. 111-120, 2007.

[15] S.-Y. Hao, S.-S. Xie, and S.-C. Yi, "The Galerkin method for the KdV equation using a new basis of smooth piecewise cubic polynomials," Applied Mathematics and Computation, vol. 218, no. 17, pp. 8659-8671, 2012.

[16] G. Lin, L. Grinberg, and G. E. Karniadakis, "Numerical studies of the stochastic Korteweg-de Vries equation," Journal of Computational Physics, vol. 213, no. 2, pp. 676-703, 2006.

[17] R.-X. Liu and L.-L. Wu, "Small-stencil Padé schemes to solve nonlinear evolution equations," Applied Mathematics and Mechanics, vol. 26, no. 7, pp. 872-881, 2005.

[18] R.-G. Yu, R.-H. Wang, and C.-G. Zhu, "A numerical method for solving $\mathrm{KdV}$ equation with multilevel B-spline quasiinterpolation," Applicable Analysis, vol. 92, no. 8, pp. 1682-1690, 2013.

[19] H. Takewaki, A. Nishiguchi, and T. Yabe, "Cubic interpolated pseudoparticle method (CIP) for solving hyperbolic-type equations," Journal of Computational Physics, vol. 61, no. 2, pp. 261268, 1985.

[20] H. Takewaki and T. Yabe, "The cubic-interpolated pseudo particle (CIP) method: application to nonlinear and multidimensional hyperbolic equations," Journal of Computational Physics, vol. 70, no. 2, pp. 355-372, 1987.

[21] T. Yabe and T. Aoki, "A universal solver for hyperbolic equations by cubic-polynomial interpolation. I. One-dimensional solver," Computer Physics Communications, vol. 66, no. 2-3, pp. 219-232, 1991.

[22] T. Ishikawa, T. Yabe, P. Y. Wang, T. Aoki, and F. Ikeda, "A universal solver for hyperbolicequations by cubic-polynomial interpolation. 2. 2-dimensional and 3-dimensional solvers," Computer Physics Communications, vol. 66, no. 2-3, pp. 233242, 1991.

[23] S. K. Lele, "Compact finite difference schemes with spectral-like resolution," Journal of Computational Physics, vol. 103, no. 1, pp. 16-42, 1992.

[24] J. Li and M. R. Visbal, "High-order compact schemes for nonlinear dispersive waves," Journal of Scientific Computing, vol. 26, no. 1, pp. 1-23, 2006.

[25] M. M. Hassan, "Exact solitary wave solutions for a generalized KdV-Burgers equation," Chaos, Solitons and Fractals, vol. 19, no. 5, pp. 1201-1206, 2004.

[26] T. Yabe, R. Tanaka, T. Nakamura, and F. Xiao, "An exactly conservative semi-lagrangian scheme (CIP-CSL) in one dimension," Monthly Weather Review, vol. 129, no. 2, pp. 332-344, 2001.

[27] D. V. Gaitonde and M. R. Visbal, "High-order schemes for navier-stokes equations: algorithm and implementation into fdl3di," Tech. Rep., DTIC Document, 1998.

[28] R. M. Miura, C. S. Gardner, and M. D. Kruskal, "Kortewegde Vries equation and generalizations: II. Existence of conservation laws and constants of motion," Journal of Mathematical Physics, vol. 9, pp. 1204-1209, 1968.

[29] M. A. Helal and M. S. Mehanna, "A comparative study between two different methods for solving the general Korteweg-de Vries equation (GKdV)," Chaos, Solitons and Fractals, vol. 33, no. 3, pp. 725-739, 2007.

[30] H. N. A. Ismail, K. R. Raslan, and G. S. E. Salem, "Solitary wave solutions for the general KDV equation by Adomian decomposition method," Applied Mathematics and Computation, vol. 154, no. 1, pp. 17-29, 2004. 


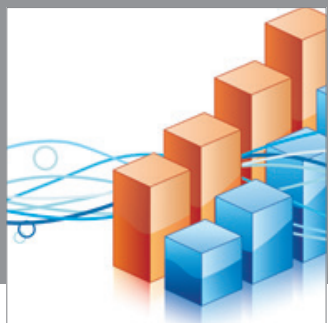

Advances in

Operations Research

mansans

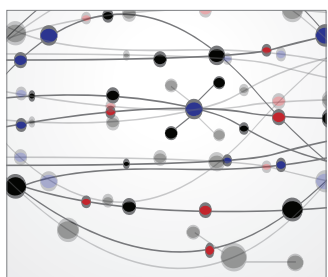

The Scientific World Journal
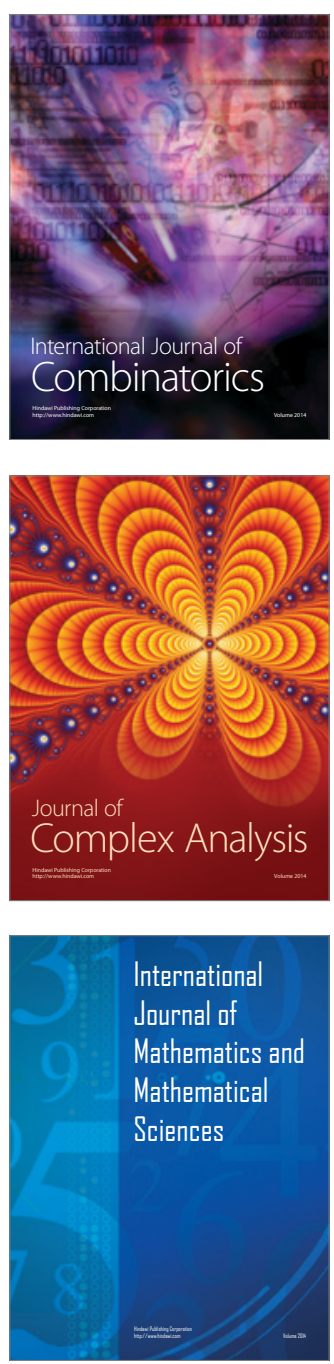
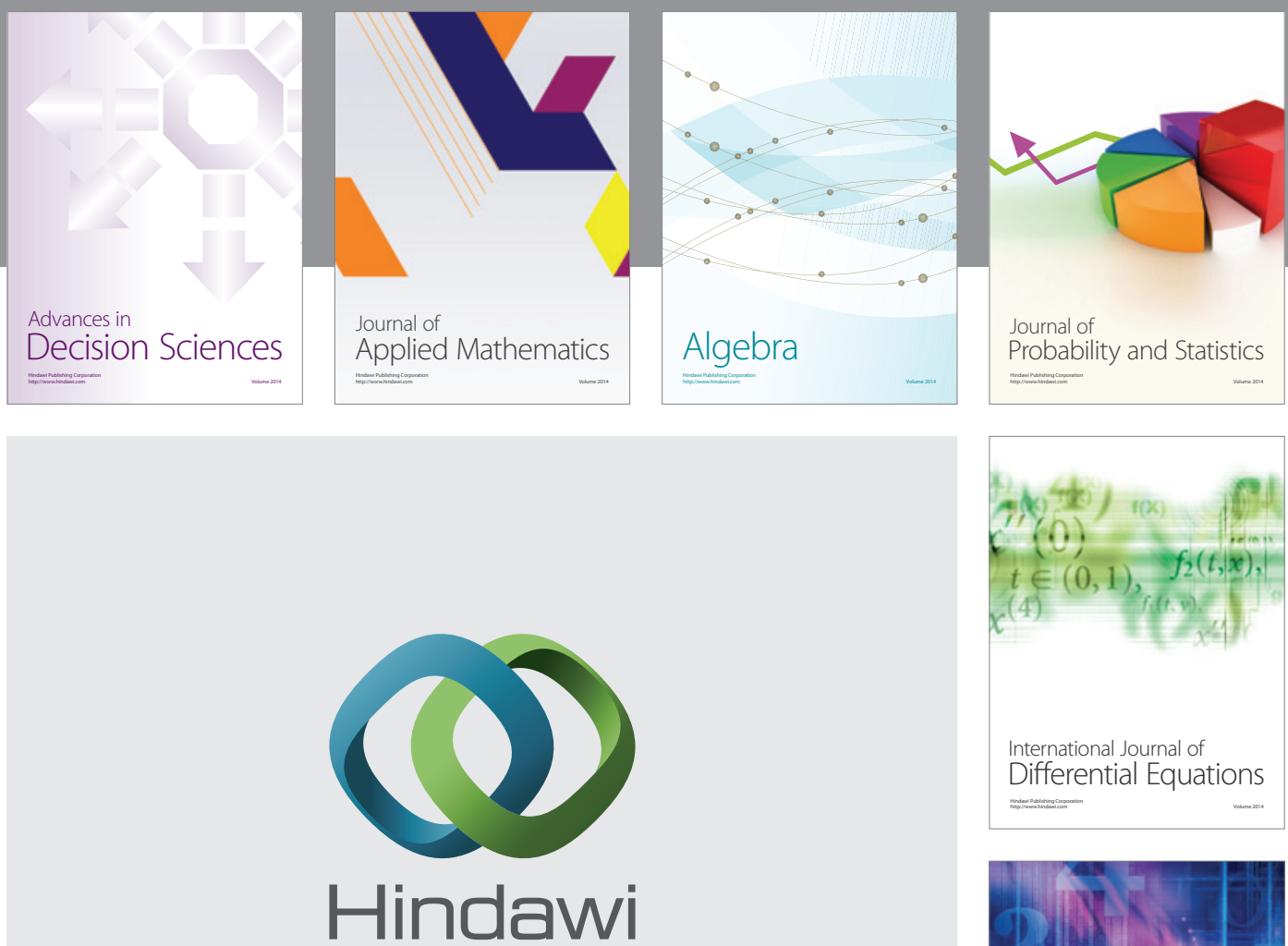

Submit your manuscripts at http://www.hindawi.com
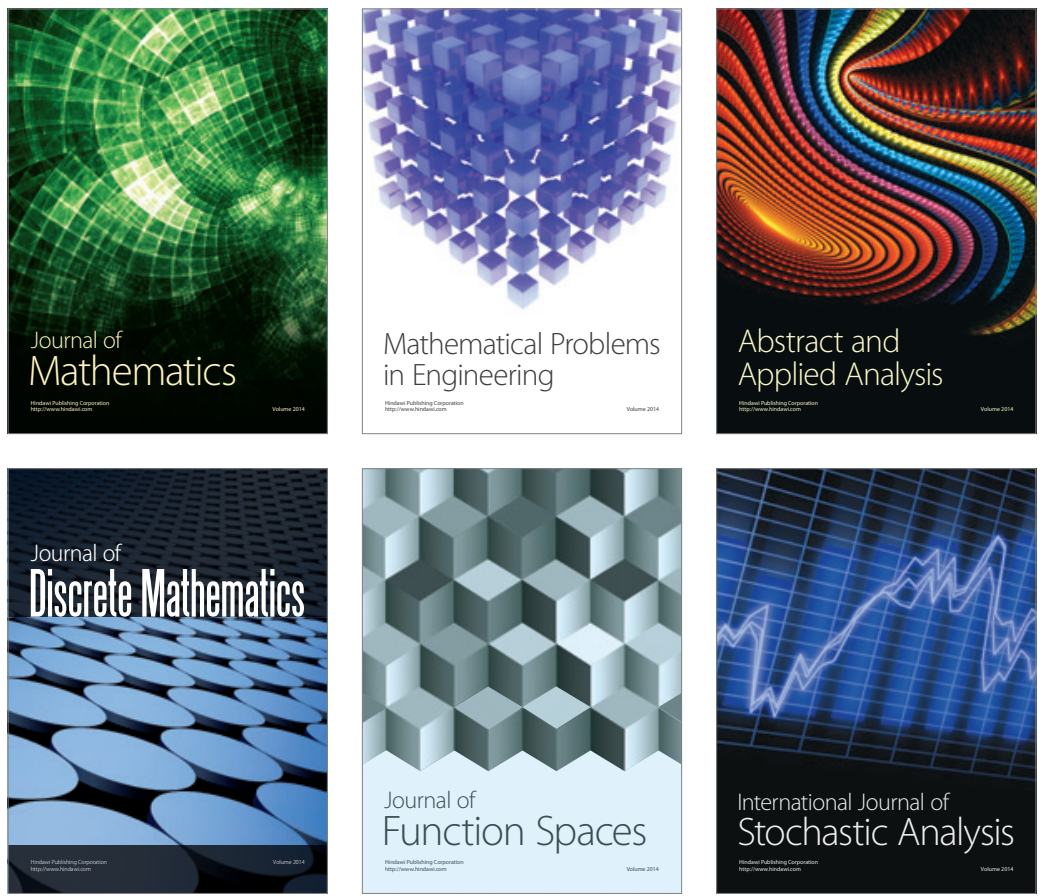

Journal of

Function Spaces

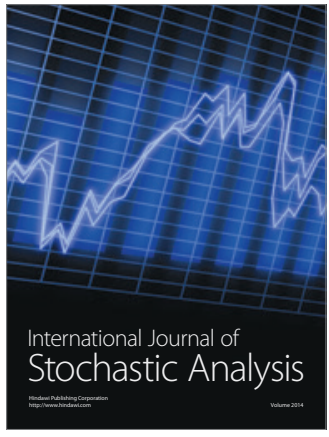

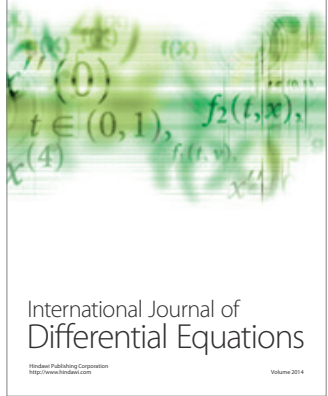
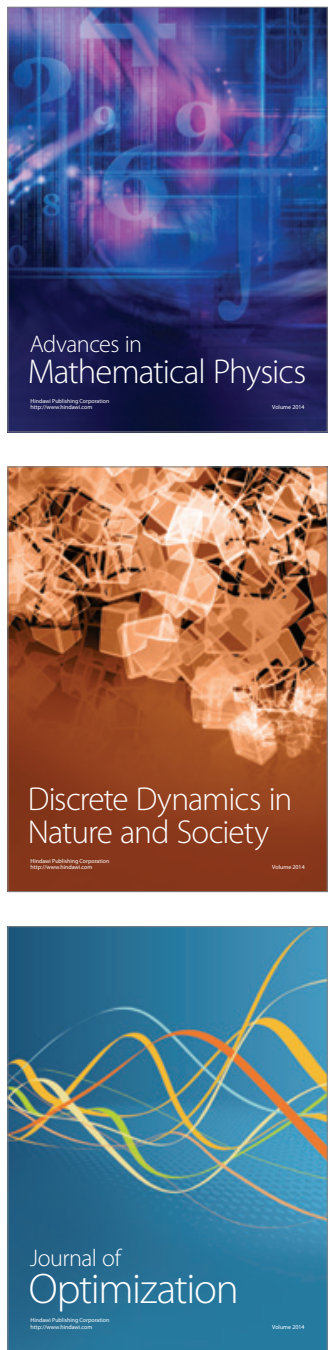